\title{
Thermally excited spin current and giant magnetothermopower in metals with embedded ferromagnetic nanoclusters
}

\author{
Oleksandr Tsyplyatyev, Oleksiy Kashuba, and Vladimir I. Fal'ko \\ Physics Department, Lancaster University, Lancaster LA1 4YB, United Kingdom
}

(Received 1 September 2006; published 11 October 2006)

\begin{abstract}
We show that a thermally excited spin current naturally appears in metals with embedded ferromagnetic nanoclusters. When such materials are subjected to a magnetic field, a spin current can be generated by a temperature gradient across the sample as a signature of electron-hole symmetry breaking in a metal due to the electron spin-flip scattering from polarized magnetic moments. Such a spin current can be observed via a giant magnetothermopower which tracks the polarization state of the magnetic subsystem and is proportional to the magnetoresistance. Our theory explains the recent experiment on Co clusters in copper by S. Serrano-Guisan et al. [Nat. Mater. 5, 730 (2006)].
\end{abstract}

DOI: 10.1103/PhysRevB.74.132403

PACS number(s): 75.75.+a, 72.25.Ba, 72.15.Jf, 73.22.-f

Theoretical studies of spin currents in metals and semiconductors have recently been fuelled by the search for materials to develop spintronic devices. ${ }^{1}$ Among the materials studied of particular interest had been composite ferromagnetic and nonmagnetic metallic systems, such as metallic multilayers $^{2}$ and metals with embedded ferromagnetic nanoclusters, ${ }^{3}$ where the electronic transport strongly depends on the electron spin leading to the effect of the giant magnetoresistance (MR).

In metals with ferromagnetic nanoclusters ${ }^{3,4}$ or magnetic impurities ${ }^{5}$ with a large spin, $s \gg 1$, the magnetoresistance reflects the degree of polarization, $\left\langle l_{z}\right\rangle=\operatorname{coth}(y)-y^{-1}, y$ $=\mu B s / k T$ of a magnetic subsystem by a magnetic field $\mathbf{B}$ $=\mathbf{n}_{z} B$,

$$
\Delta(B) \equiv \frac{R(B)}{R(0)}-1=-\frac{\left(\tau_{\uparrow}-\tau_{\downarrow}\right)^{2}}{4 \tau^{2}}\left\langle l_{z}\right\rangle^{2} .
$$

The MR effect develops across the magnetic field range $B_{M R} \sim k T / \mu s$, and its strength depends on the relative difference between the mean free path time $\tau_{\uparrow(\downarrow)}$ of electrons with spins parallel (antiparallel) to the cluster polarization axis. Here, $\tau_{\uparrow(\downarrow)}$ is defined as the mean free path in a metal with magnetic clusters whose spin polarizations are fully up(down). The Fermi density of states per spin, $\gamma$, is identical for both electron spin orientations, and $\tau=\frac{1}{2}\left(\tau_{\uparrow}+\tau_{\downarrow}\right)$ is a characteristic mean free path time, while $\mu$ is the Bohr magneton.

In this Brief Report we show that a thermally excited spin current naturally appears in metals with embedded ferromagnetic nanoclusters (FMNCs) when subjected to a magnetic field and temperature gradient. This effect appears through the manifestation of electron-hole symmetry breaking via electron spin-flip scattering from polarized magnetic moments. When two parts of a metallic sample with embedded FMNCs are held at different temperatures $T_{1}>T_{2}$, the thermally equilibrating heat flux must be accompanied by a transport of magnetization, ${ }^{6-8}$ i.e., a spin current, $\mathbf{j}_{s}$. This spin current acts to equilibrate the cluster polarization on opposing sides of the temperature gradient. Locally, the equilibration process requires spin transfer from clusters to conduction electrons via spin-flip scattering at a rate $\tau_{s}^{-1}$, which is the inverse mean free path time of the spin-flip scattering process [formally defined latter, Eq. (6)]. After a single spin-flip scattering event, the scattered electron will carry the transferred magnetization while diffusing (with diffusion coefficient $D$ ) between different parts of the sample held at different temperatures, thus leading to a spin current,

$$
\mathbf{j}_{s}=\beta \nabla T, \quad \beta \approx \frac{1}{2} \hbar k \gamma D \frac{\tau f}{\tau_{s}}\left\langle l_{z}\right\rangle .
$$

Here $f=\frac{x^{2} e^{x}}{\left(e^{x}-1\right)^{2}}$ and $x=\mu B / k T$ show that the spin current will persist up to a value of the magnetic field $B_{s} \sim k T / \mu$, which is much larger than the typical field value at which MR develops, $B_{M R} \ll B_{s}$. Additionally, due to the difference in the mean free path times of "up" and "down" spin carriers, $\left(\tau_{\uparrow}\right.$ $\left.-\tau_{\downarrow}\right)\left\langle l_{z}\right\rangle$, the spin current "drags" a charge current, $\mathbf{j} \propto \chi \nabla T$. In an open circuit, this generates a thermopower, $V_{12} \propto c(B)$ $\left[T_{1}-T_{2}\right]$, with a strong magnetic field dependence resembling (for $B<k T / \mu$ ) that of MR,

$$
\Xi(B) \equiv c(B)-c(0) \approx \frac{k}{e} \frac{\tau^{2} f}{\tau_{s}\left(\tau_{\downarrow}-\tau_{\uparrow}\right)} \Delta(B) .
$$

A microscopic justification of the above-presented phenomenological argument is supported by the following analysis of the electron-hole asymmetry in a composite metal with ferromagnetic components. In normal metals, the electron-hole asymmetry leading to the thermopower is caused by the energy dependence of the density of states of electrons near the Fermi level. In materials containing a polarized magnetic subsystem, the electron-hole asymmetry is created in an alternative way-via the energy and spin dependence of a quasiparticle scattering rate. ${ }^{6-8}$ In the system we discuss in this Brief Report, the formation of kinetic electron-hole asymmetry can be illustrated using a Kondotype model $^{11}$ which treats a FMNC as an impurity with a large spin, $s$, and contains all the necessary ingredients to describe the MR effect simultaneously with the generation of spin current and magnetothermopower.

Thus we model the FMNCs by the Hamiltonian 
(a) $\because \because, \dot{U} \sim G^{A}$

(b)
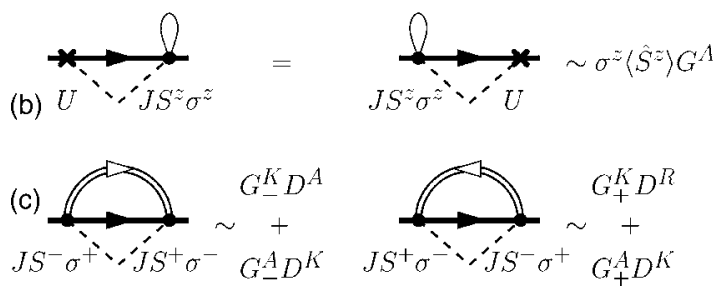

FIG. 1. Self-energy diagrams: thick solid line is free electrons Green's function, $G_{ \pm}=G(\varepsilon \pm \mu B)$, thin dashed line is impurity line, thin solid line connected both ends to a single vertex is an average of spin operator, double thin solid line connected to two vertices is the spin-spin correlator, cross is the potential vertex $U$, and dot is the exchange vertex $J$.

$$
-\mu B \hat{s}_{z}+\int \psi^{\dagger}(\mathbf{r}) \sum_{i}(U+J \hat{\sigma} \cdot \hat{\mathbf{s}}) \delta\left(\mathbf{r}-\mathbf{r}_{i}\right) \psi(\mathbf{r}) d^{3} \mathbf{r}
$$

Here, the potential $U$ accounts for the FNMC charge and for band mismatch between normal and magnetic metals, whereas $J$ is the exchange interaction; $\mu s$ is the magnetic moment of each cluster; and $\sigma$ stands for the electron spin operator. The MR analysis in materials with large spin clusters $s \gg 1$ can be done using a static exchange field model, ${ }^{3}$ where the operator $\hat{\mathbf{s}}$ is replaced by $\mathbf{s} \mathbf{l}$ (here $\mathbf{l}$ is a unit vector in the direction of polarization of an individual cluster). In contrast, the analysis of a thermally excited spin current and the magnetothermopower needs to take into account the quantum nature of the cluster spin, manifested through the electron spin-flip process.

The formation of electron-hole asymmetry in the quasiparticle lifetime becomes apparent after analyzing the imaginary part of the self-energy to the lowest nonvanishing order of perturbation theory. Figure 1 shows the Keldysh diagrams which appear in the self-energy $\hat{\Sigma}^{A(R)}$ in the second order of the electron-cluster interaction, after averaging over random positions, $\mathbf{r}_{i}$, of the clusters. We assume a thermal distribution of cluster spin polarization, with temperature $T$. The diagrams in Fig. 1 incorporate two types of vertices: crosses stand for the potential $U$ and dots for the exchange coupling $J$. Dashed lines represent impurity averaging $\left[--=n_{c} \delta(\mathbf{r}\right.$ $\left.\left.-\mathbf{r}^{\prime}\right)\right]$. Thin solid lines indicate the thermodynamic average and dynamical correlators of the cluster spin: a loop stands for the thermodynamic average $\left\langle s_{z}\right\rangle$, a double line without an arrow is $\left\langle\left(s_{z}\right)^{2}\right\rangle$, whereas a thin double line with arrows stands for the free-spin Green functions, ${ }^{10} D^{R / A}(t)$ $=\mp i\left\langle\left[\hat{s}^{+}(t) \hat{s}^{-}(0)\right]_{-}\right\rangle \theta( \pm t)$ and $D^{K}(t)=-i\left\langle\left\{\hat{s}^{+}(t) \hat{s}^{-}(0)\right\}_{+}\right\rangle$, where $\hat{s}^{ \pm}=\hat{s}_{x} \pm i \hat{s}_{y}$. In the energy representation,

$$
\begin{gathered}
=\triangleright=D^{R / A}(\omega)=\frac{2\left\langle s_{z}\right\rangle}{\omega-\mu B \pm i \delta}, \\
D^{K}(\omega)=\operatorname{coth} \frac{\omega}{2 T}\left(D^{R}(\omega)-D^{A}(\omega)\right) .
\end{gathered}
$$

Although Wick's theorem cannot be applied to spin operators in higher orders of perturbation theory, this does not affect the analysis of the diagrams in the Born approximation. Finally, bold solid lines with arrows are free electronic Green functions,

$$
\begin{gathered}
-=G^{R / A}(\varepsilon, \mathbf{p})=\frac{1}{\varepsilon-\xi_{\mathbf{p}} \pm i \delta}, \\
G^{K}(\varepsilon, \mathbf{p})=\tanh \frac{\varepsilon}{2 T}\left(G^{R}(\varepsilon, \mathbf{p})-G^{A}(\varepsilon, \mathbf{p})\right),
\end{gathered}
$$

where the electron energy $\varepsilon$ is determined with respect to the Fermi level.

The diagram in Fig. 1(a) describes scattering from a scalar potential, whereas diagrams (b) and (d) only include the exchange interaction. The interference between the scalar and exchange potential scattering amplitudes (from the same impurity) is taken into account by the diagrams in Fig. 1(c), which is responsible for the spin-dependence of the electron mean free path in the presence of polarized clusters. Together, these diagrams yield ${ }^{11,12}$

$$
\begin{aligned}
\operatorname{Im} \hat{\Sigma}^{A}(\varepsilon)= & \pi \gamma n_{c}\left[U^{2}+J^{2} s(s+1)+2 U J\left\langle s_{z}\right\rangle \hat{\sigma}_{z}\right] \\
& -\pi \gamma n_{c} J^{2}\left\langle s_{z}\right\rangle \hat{\sigma}_{z} \tanh \left(\frac{\varepsilon+\mu B \hat{\sigma}_{z}}{2 T}\right),
\end{aligned}
$$

where $n_{c}$ is the concentration of FMNCs. Diagrams 1(a)-1(c) describe the elastic processes. The spin-flip diagrams 1(d) contain an inelastic part resulting in the energy-dependent contribution towards $\operatorname{Im} \hat{\Sigma}^{A}$ in Eq. (5), and they are responsible for the kinetic electron-hole asymmetry. This asymmetry is most pronounced when the ensemble of clusters is fully polarized, $\left\langle l_{z}\right\rangle \rightarrow 1$, and vanishes when $\left\langle l_{z}\right\rangle=0$.

To explain the origin of this asymmetry and its polarization dependence we consider the limit of $\left\langle l_{z}\right\rangle \rightarrow 1$, so that all FMNCs are polarized "up." Figure 2(a) shows that an incident spin- $\downarrow$ electron with energy $\epsilon>\mu B$ above the Fermi level is able to flip its spin turning the spin of FMNC from the polarization axis. The amplitude of such a process is $A$ $\sim J\left\langle s-1\left|\hat{s}^{-}\right| s\right\rangle \sim J \sqrt{s}$, which results in the scattering rate $\tau_{s}^{-1} \propto J^{2} s$. The relevant range of a quasiparticle excitation energy for this process is set by the FMNC energy splitting, $\mu B$, between its initial and scattered spin state, which differ by $s_{z}-s_{z}^{\prime}=1$. A similar process is possible for the incident spin- $\uparrow$ hole with $\epsilon<-\mu B$ below the Fermi level, Fig. 2(d): it corresponds to the reverse process of an equilibrium spin- $\downarrow$ electron relaxing into an empty state with opposite spin below the Fermi level (hole). In contrast, neither spin- $\uparrow$ electrons above the Fermi level, nor spin- $\downarrow$ holes shown in Figs. 2(b) and 2(c) can scatter with changing spin state, since they cannot increase further the maximal $s_{z}=s$ of a cluster. All together, these processes determine the energy-dependent part in the electron scattering rate from fully polarized clusters,

$$
\operatorname{Im} \hat{\Sigma}_{\left\langle l_{z}\right\rangle \rightarrow 1}^{A}=\left(\begin{array}{cc}
\frac{\tau_{\uparrow}^{-1}+\theta(-\varepsilon-\mu B) \tau_{s}^{-1}}{2} & 0 \\
0 & \frac{\tau_{\downarrow}^{-1}+\theta(\varepsilon-\mu B) \tau_{s}^{-1}}{2}
\end{array}\right),
$$



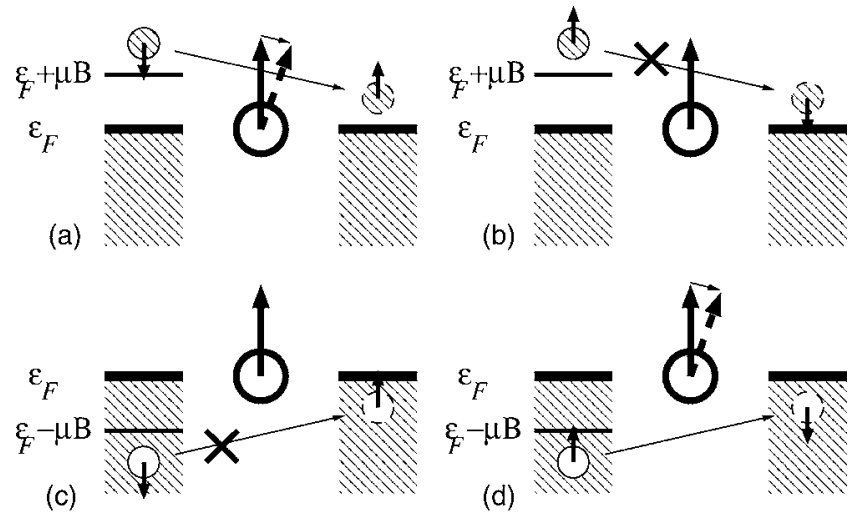

(d)

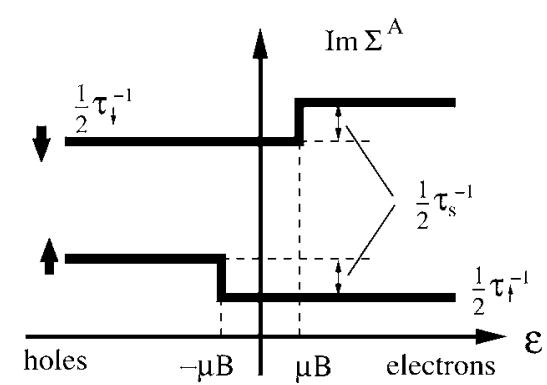

(e)

FIG. 2. Schematic representation of allowed and forbidden processes leading to the energy and spin dependent quasiparticles relaxation rate (a) and (d) allowed processes, (b) and (c) forbidden processes, and (e) dependence of $\operatorname{Im} \Sigma^{A}$ as a function of energy for different spin directions (graph is drawn for the case of $J U<0$ ).

$$
\begin{gathered}
\tau_{\uparrow(\downarrow)}^{-1}=\frac{2 \pi n_{c} \gamma}{\hbar}\left[U^{2}+J^{2} s(s+1) \pm 2 U J s\right], \\
\tau_{s}^{-1}=\frac{4 \pi n_{c} \gamma}{\hbar} J^{2} s .
\end{gathered}
$$

Note that for a cluster with $N$ magnetic atoms $S \propto N$ and $U$ $\propto N$ so that $\tau_{\uparrow(\downarrow)}^{-1} \propto N^{2}$, whereas $\tau_{s}^{-1} \propto N$. The resulting behavior of the quasiparticle lifetime for electrons in the presence of polarized FMNCs is sketched in Fig. 2(e). It indicates that the electron-hole asymmetry is inverted in the opposite spin channels, in accordance with the symmetry of the Kondo problem. $^{13}$

The inverted electron-hole asymmetry in the opposite spin channel results in the generation of a spin current when two parts of the system are held at different temperatures. To describe a thermally generated spin current, $\mathbf{j}_{s}=\beta \nabla T$, as well as the electric current $\mathbf{j}=\chi \nabla T$, we use the kinetic equation approach. That is, we study a steady-state kinetic equation,

$$
\left[\mathbf{v} \cdot \nabla-e \mathbf{E} \cdot \partial_{\mathbf{p}}\right] \varrho_{ \pm}=\left\langle I_{ \pm}\left(\hat{\varrho}, s_{z}\right)\right\rangle
$$

where the collision term $I_{ \pm}\left(\hat{\varrho}, s_{z}\right)$ describes the balance in the distribution functions $\varrho_{+}$of spin- $\uparrow$ and $\varrho_{-}$of spin- $\downarrow$ electrons which scatter from a group of FMNCs in a given initial spin state, $s_{z}$. The brackets $\langle\cdots\rangle$ stand for averaging over the thermal distribution of cluster spins. The averaged collision term therefore takes into account all electron scattering processes described by the diagrams in Fig. 1,

$$
\begin{aligned}
I_{ \pm}= & \frac{2 \pi n_{c}\left|U \pm J s_{z}\right|^{2}}{\hbar} \int \frac{d^{3} \mathbf{p}^{\prime}}{h^{3}}\left(\varrho_{ \pm}^{\prime}-\varrho_{ \pm}\right) \delta\left(\epsilon_{\mathbf{p}}-\epsilon_{\mathbf{p}^{\prime}}\right) \\
& +\frac{2 \pi n_{c} J^{2}}{\hbar} \int \frac{d^{3} \mathbf{p}^{\prime}}{h^{3}}\left[\left(s^{2}-s_{z}^{2}+s \pm s_{z}\right) \varrho_{\mp}^{\prime}\left(1-\varrho_{ \pm}\right)\right. \\
& \left.-\left(s^{2}-s_{z}^{2}+s \mp s_{z}\right) \varrho_{ \pm}\left(1-\varrho_{\mp}^{\prime}\right)\right] \delta\left(\epsilon_{\mathbf{p}^{\prime}}-\epsilon_{\mathbf{p}} \mp \mu B\right) .
\end{aligned}
$$

The first term in $I_{ \pm}$describes the elastic spin-conserving processes in Figs. 1(a)-1(c), whereas the second takes care of spin-flip processes corresponding to diagrams in Fig. 1(d). By inspection, one can see that the probabilities of electrons $\downarrow \rightarrow \uparrow$ and $\uparrow \rightarrow \downarrow$ spin-flip scattering from the same cluster with a given $s_{z}$ slightly differ. In a system where FMNCs are partially polarized (along the external magnetic field $\mathbf{B}$ $\left.=\mathbf{n}_{z} B\right)$ this leads to the energy- and spin-dependent electron momentum relaxation rate, $2 \operatorname{Im} \Sigma_{\alpha \alpha}^{A} / \hbar$ (where $\alpha=\uparrow, \downarrow$ ) given by Eq. (5). In the leading order in a small temperature gradient $\left(v_{F} \tau T^{-1} \nabla T \ll 1\right)$, the spin-current $\mathbf{j}_{s}$ $=\frac{\hbar}{6} \int \gamma d \varepsilon \operatorname{Tr}\left\{\hat{\sigma}_{z} \hat{\boldsymbol{\rho}}\right\}$ and electric current $\mathbf{j}=\frac{e}{3} \int \gamma d \varepsilon \operatorname{Tr}\{\hat{\boldsymbol{\rho}}\}$ are determined by the first angular harmonic in momentum space, $\hat{\boldsymbol{\rho}}$ of the electron spin-density matrix, $\hat{\varrho} \approx \varrho_{T}+\hat{\boldsymbol{\rho}} \cdot \mathbf{p} / p$. This first angular harmonic can be found using the equation

$$
\frac{2}{\hbar} \operatorname{Im} \hat{\Sigma}^{A} \hat{\boldsymbol{\rho}}=\frac{\varepsilon v_{F} \partial_{\varepsilon} \varrho_{T}}{T} \nabla T
$$

where $\varrho_{T}(\varepsilon)=\left[e^{\varepsilon / k T}+1\right]^{-1}$ is the Fermi function.

As a result, we calculate the spin current, $\mathbf{j}_{s}=\beta \nabla T$, which, for large spin clusters, $s \gg 1$ is described by

$$
\beta=-\frac{2 \hbar k \gamma v_{F}^{2}}{3} \frac{\tau_{\uparrow}^{2} \tau_{\downarrow}^{2} f}{\tau_{s}}\left\langle l_{z}\right\rangle \frac{\left(\tau_{\uparrow}+\tau_{\downarrow}\right)^{2}+\left(\tau_{\uparrow}-\tau_{\downarrow}\right)^{2}\left\langle l_{z}\right\rangle^{2}}{\left[\left(\tau_{\uparrow}+\tau_{\downarrow}\right)^{2}-\left(\tau_{\uparrow}-\tau_{\downarrow}\right)^{2}\left\langle l_{z}\right\rangle^{2}\right]^{2}} .
$$

In the above result, scattering rates $\tau_{\uparrow(\downarrow)}^{-1}$ and $\tau_{s}^{-1}$ are defined in Eq. (6), and the factor $f=\frac{x^{2} e^{x}}{\left(e^{x}-1\right)^{2}}$ with $x=\mu B / k T$ is specified for the case $s \gg 1$ and takes into account the suppression of the spin transfer rate at low temperatures and high magnetic field, such that $\mu B>k T$, since it requires the electron energy transfer $\epsilon-\epsilon^{\prime}=\mu B$. Also, in most of the metals with embedded ferromagnetic clusters, the maximum MR effect is $\Delta$ $\lesssim 10 \%$, which is additionally suppressed by nonmagnetic impurities and phonon scattering. Therefore we can simplify the result in Eq. (8) further, using the fact that $\left(\tau_{\uparrow}-\tau_{\downarrow}\right) /$ $\left(\tau_{\uparrow}+\tau_{\downarrow}\right) \ll 1$, which leads to the approximate form in Eq. (1).

Due to the difference, $\left(\tau_{\uparrow}-\tau_{\downarrow}\right)\left\langle l_{z}\right\rangle$ between the mean free path times of spin- $\uparrow$ and $-\downarrow$ carriers scattering from the ensemble of partially polarized FMNCs, the spin-current "drags" a charge current $\mathbf{j} \propto \varkappa \nabla T$. For $s \gg 1$, we find

$$
\varkappa=\frac{8 e v_{F}^{2} \gamma k}{3 \tau_{s}} \frac{\tau_{\uparrow}^{2} \tau_{\downarrow}^{2}\left(\tau_{\uparrow}-\tau_{\downarrow}\right)\left\langle l_{z}\right\rangle^{2} f\left(\frac{\mu B}{k T}\right)}{\left[\left(\tau_{\uparrow}+\tau_{\downarrow}\right)^{2}-\left(\tau_{\uparrow}-\tau_{\downarrow}\right)^{2}\left\langle l_{z}\right\rangle^{2}\right]^{2}} .
$$

Together with the MR of this material obtained using the same approximations, 


$$
R=\frac{3}{e^{2} v_{F}^{2} \gamma} \frac{\tau_{\uparrow}+\tau_{\downarrow}}{4 \tau_{\uparrow} \tau_{\downarrow}}\left[1-\frac{\left(\tau_{\uparrow}-\tau_{\downarrow}\right)^{2}}{\left(\tau_{\uparrow}+\tau_{\downarrow}\right)^{2}}\left\langle l_{z}\right\rangle^{2}\right],
$$

this determines the thermopower coefficient $c=-\varkappa R$ with a magnetic field dependence. The magnetothermopower $\Xi(B) \equiv c(B)-c(0)$ is related to the observable MR as

$$
\Xi(B)=\frac{k}{e} \frac{\tau_{\downarrow} \tau_{\uparrow}}{\tau_{s}\left(\tau_{\downarrow}-\tau_{\uparrow}\right)} \frac{\Delta(B)}{1+\Delta(B)} f\left(\frac{\mu B}{k T}\right) .
$$

The magnetic field dependence of $\Xi(B)$, implicit in the above result, contains two field scales. ${ }^{14}$ At a low magnetic field where the polarization of clusters develops, the MTP is proportional to the MR and saturates together with $\Delta(B)$ at the field $B_{\mathrm{MR}}$. At a higher field range, $B>k T / \mu$ the MTP is suppressed and dies away. For weak MR materials, $\Delta \lesssim 0.1$, where $\tau_{\downarrow}-\tau_{\uparrow} \ll \tau=\frac{1}{2}\left(\tau_{\downarrow}+\tau_{\uparrow}\right)$, the above relation can be approximated using the formula in Eq. (2).

At the early stage of study of Kondo impurity in metals ${ }^{13}$ a strong magnetothermopower has been noticed in various dilute magnetic alloys. ${ }^{15-18}$ Later, a strong magnetic field dependent thermopower has been observed in metals with embedded ferromagnetic nanoclusters, ${ }^{19-23}$ where it has been attributed to the energy dependent density of states in a weakly ferromagnetic metal with a complex band structure. ${ }^{9}$ However, when viewed in terms of the above-presented theory, these observations indicate the presence of thermally excited spin current, Eqs. (1) and (8) generated by a temperature gradient.

Recently, a giant MTP has been measured in copper with embedded Co clusters of a controlled size. It was noticed that MTP weakens with an increase of the number of atoms, $N$, in a FMNC. ${ }^{24}$ This behavior as well as the observed direct correspondence between MTP and MR can be explained on the basis of the result in Eq. (9). The matter is that for a cluster with $N$ magnetic atoms $\tau_{\uparrow(\downarrow)}^{-1} \propto N^{2}$, whereas $\tau_{s}^{-1} \propto N$. Therefore for two samples with the same densities of the embedded clusters containing $N$ and $N^{\prime}$ atoms, we estimate $\Xi(N) / \Xi\left(N^{\prime}\right) \propto N^{\prime} / N$, the MTP is weaker in the system with larger clusters.

The authors thank B. Altshuler, G. Bauer, C. Marrows, L. Glazman, A. Guenault, E. McCann, and J. Jefferson for discussions. This work was funded by the EU STREP NPM2CT-2003-505587 "SFINX" and the Lancaster-EPSRC Portfolio Partnership EP/C511743.
${ }^{1}$ I. Žutić, J. Fabian, and S. Das Sarma, Rev. Mod. Phys. 76, 323 (2004); G. A. Prinz, Science 282, 1660 (1998), and references therein.

${ }^{2}$ T. Valet and A. Fert, Phys. Rev. B 48, 7099 (1993); D. H. Mosca et al., J. Magn. Magn. Mater. 94, L1 (1991).

${ }^{3}$ S. Zhang, Appl. Phys. Lett. 61, 1855 (1992); S. Zhang and P. M. Levy, J. Appl. Phys. 73, 5315 (1993); H. Camlong, S. Zhang, and P. M. Levy, ibid. 75, 6906 (1994); P. Holody, L. B. Steren, R. Morel, A. Fert, R. Loloee, and P. A. Schroeder, Phys. Rev. B 50, 12999 (1994).

${ }^{4}$ A. E. Berkowitz, J. R. Mitchell, M J. Carey, A. P. Young, S. Zhang, F. E. Spada, F. T. Parker, A. Hutten, and G. Thomas, Phys. Rev. Lett. 68, 3745 (1992); J. Xiao, J. S. Jiang, and C. L. Chien, Phys. Rev. B 46, 9266 (1992).

${ }^{5}$ K. Yosida, Phys. Rev. 107, 396 (1957); H. Rohrer, ibid. 174, 583 (1968).

${ }^{6}$ I. Korenblit, J. Phys. F: Met. Phys. 12, 1259 (1982).

${ }^{7}$ L. Piraux et al., J. Magn. Magn. Mater. 110, L247 (1992); J. L. Duval et al., J. Appl. Phys. 75, 7070 (1994).

${ }^{8}$ E. McCann and V. I. Fal'ko, Appl. Phys. Lett. 81, 3609 (2002); E. McCann and V. I. Fal'ko, Phys. Rev. B 66, 134424 (2002).

${ }^{9}$ L. Xing, Y. C. Chang, M. B. Salamon, D. M. Frenkel, J. Shi, and J. P. Lu, Phys. Rev. B 48, 6728 (1993).

${ }^{10}$ B. Giovannini and S. Koide, Prog. Theor. Phys. 34, 705 (1965).

${ }^{11}$ J. Kondo, Prog. Theor. Phys. 34, 372 (1965); L. Gurevich and I. Yassievich, Sov. Phys. JETP 20, 922 (1965); K. Maki, Prog. Theor. Phys. 41, 586 (1969); R. Weiner and M. Beal-Monod, Phys. Rev. B 2, 2675 (1970); N. Kawakami, T. Usuki, and A. Okiji, J. Phys. Soc. Jpn. 56, 1539 (1987).
${ }^{12}$ M. G. Vavilov, L. I. Glazman, and A. I. Larkin, Phys. Rev. B 68 , 075119 (2003).

${ }^{13}$ A. Hewson, The Kondo Problem to Heavy Fermions (Cambridge University Press, Cambridge, 1993).

${ }^{14}$ For a metal with spin- $\frac{1}{2}$ impurities, their polarization and MR develop over the same range of a magnetic field as the field suppressing the electron spin-flip scattering. Therefore in a metal with spin- $\frac{1}{2}$ Anderson impurities, magnetothermopower would display a sharp nonmonotonic field dependence with a maximum effect at $B \approx 2 k T / \mu, \Xi=\frac{k}{e} \frac{\tau_{\downarrow} \tau_{\uparrow}}{\tau_{s}\left(\tau_{\downarrow}-\tau_{\uparrow}\right)} \frac{\Delta(B)}{\cos h^{2}(\mu B / 2 k T)}$

${ }^{15}$ D. Huntley and C. Walker, Can. J. Phys. 47, 805 (1969).

${ }^{16}$ R. Berman et al., Phys. Lett. 27A, 464 (1968); R. Berman and J. Kopp, J. Phys. F: Met. Phys. 1, 457 (1971); J. Kopp, ibid. 6, 1211 (1975).

${ }^{17}$ M. Read and A. Guenault, J. Phys. F: Met. Phys. 4, 94 (1974).

${ }^{18}$ L. J. Azevedo, P. M. Chaikin, W. G. Clark, W. W. Fuller, and J. Hammann, Phys. Rev. B 20, 4450 (1979).

${ }^{19}$ J. Shi, E. Kita, L. Xing, and M. Salamon, Phys. Rev. B 48, 16119 (1993); J. Shi, K. Pettit, E. Kita, S. S. P. Parkin, R. Nakatani, and M. B. Salamon, ibid. 54, 15273 (1996).

${ }^{20}$ L. Piraux, M. Cassart, J. S. Jiang, J. Q. Xiao, and C. L. Chien, Phys. Rev. B 48, 638 (1993).

${ }^{21}$ H. Sato et al., J. Magn. Magn. Mater. 152, 109 (1996); H. Sato, Mater. Sci. Eng., B 31, 101 (1995); H. Sato et al., J. Phys.: Condens. Matter 7, 7053 (1995).

${ }^{22}$ Y. Kobayashi et al., J. Phys.: Condens. Matter 8, 11105 (1996).

${ }^{23}$ J. Sakurai et al., J. Phys. Soc. Jpn. 66, 2240 (1997).

${ }^{24}$ S. Serrano-Guisan et al., Nat. Mater. 5, 730 (2006). 